\title{
Pico de velocidade de crescimento como alternativa para classificação maturacional associada ao desempenho motor
}

\author{
Peak height velocity as an alternative for maturational \\ classification associated with motor performance
}

\author{
Dalmo Roberto Lopes Machado 1,2 \\ Mariana Rotta Bonfim ${ }^{1,2}$ \\ Leonardo Trevizan Costa ${ }^{1,2}$
}

Resumo - A adolescência é uma fase da vida de importantes alterações físicas e maturacionais, sendo que indivíduos de mesma idade cronológica, porém mais maturados, podem apresentar vantagens esportivas em função do maior ganho de força e aumentos da massa muscular. Assim, no contexto esportivo e nas pesquisas, envolvendo crianças e adolescentes, é necessária a classificação maturacional, de forma eficiente e de fácil aplicação, auxiliando na interpretação da verdadeira relação entre a maturação e desempenho motor. Sendo assim, o objetivo do estudo foi comparar a relação entre o desempenho motor e diferentes formas de classificação da maturação biológica, envolvendo 209 rapazes de seis a 17 anos $(11,59 \pm 2,57)$, praticantes de futebol. As variáveis antropométricas foram obtidas seguindo-se os critérios estabelecidos pela ISAK. A maturação biológica foi determinada pela idade do Pico de Velocidade de Crescimento (PVC) e pela determinação da maturação sexual (genitálias e pêlos) através da auto-avaliação. O desempenho motor foi determinado por testes de sentar-e-alcançar, salto horizontal, abdominal modificado, corrida de 50 metros e corrida/caminhada de 9/12 minutos. Os resultados indicaram evolução das variáveis analisadas à medida que os indivíduos avançavam na maturação. A análise de correlação e os valores da regressão apontaram maior consistência na utilização da classificação pelo PVC como melhor explicação para o desempenho em testes motores, segundo o status maturacional, superando os valores encontrados nas comparações por idade ou maturação sexual. Desta forma, o uso do PVC como instrumento de classificação biológica passa a ser preferencialmente recomendado para jovens com características semelhantes à amostra utilizada neste estudo. Palavras-chave: Maturação; Futebol; Adolescente; Atividade motora.

Abstract - Adolescence is a phase characterized by important physical and maturational alterations, with individuals of the same chronological age, but who are more mature, may present sportive advantages because of greater force gain and additional muscle mass. Thus, in studies evaluating motor skills of children and adolescents, maturational classification should be an efficient and easily applicable tool in order to facilitate the interpretation of the true relationship between maturation and motor performance. Therefore, the objective of this study was to compare the relationship between motor performance and different types of classification of biological maturation in 209 boys aged 6 to 17 years $(11.59 \pm 2.57)$ practicing soccer. Anthropometric variables were obtained according to the ISAK criteria. Biological maturation was determined based on age at peak height velocity (PHV) and also by self-assessment of sexual maturation (genitalia and pubic hair). Motor performance was evaluated by sit-and-reach tests, horizontal jump, modified push-ups, 50-m run, and 9/12-min run/walk. The results showed progression

1 Universidade Estadual Paulista, Departamento de Educação Física, Presidente Prudente, SP. Brasil.

Recebido em 13/02/08 Aprovado em 07/04/08 in the variables analyzed as the subjects reached maturity. Correlation analysis and the regression values indicated higher consistency for the PHV classification, which better explained motor performance according to maturational status, exceeding the values obtained by comparison according to age or sexual maturation. Thus, the use of PHV as a biological classification instrument is preferably recommended for youngsters with characteristics similar to those of the present sample.

Key words: Maturation; Soccer; Adolescent; Motor activity. 


\section{INTRODUÇÃO}

A maturação biológica é um processo de importantes alterações fisiológicas que se manifestam de forma mais intensa durante a adolescência e o tempo de sua ocorrência depende do sexo e do estágio maturacional. Por conseqüência, as diferenças de performance motora nas comparações entre sujeitos de maturação adiantada com os normais ou atrasados ${ }^{1,2}$, torna necessária a classificação maturacional no contexto esportivo ou nas pesquisas realizadas com crianças e adolescentes ${ }^{2-5}$.

A opção pelo melhor instrumento deve contemplar a simplicidade e praticidade de utilização, o baixo custo operacional, a precisão das medidas, a estreita relação com o desempenho motor, o treinamento do avaliador, eliminação de fatores de interferência, entre outros, que possam apresentar distorções dos valores obti$\operatorname{dos}^{6,7}$ e, sobretudo, a ética.

A idade do pico de velocidade de crescimento (PVC) é o indicador mais comumente utilizado em estudos longitudinais, considerando a maturidade somática do adolescente ${ }^{3}$, podendo figurar numa interessante alternativa de classificação biológica. Mediante o acompanhamento das variáveis de crescimento, pode-se detectar o momento em que o indivíduo atinge o PVC. Embora seja possível determinar os picos de velocidade em estatura, peso corporal e somatório de dobras cutâneas, o marco somático mais utilizado em estudos da velocidade do crescimento é a idade do pico da estatura ${ }^{8}$.

Obviamente, seriam necessárias várias medidas durante um determinado período do crescimento, o que tornaria essa metodologia inviável para investigações transversais, quando apenas a realização de uma única medida fosse possível. Neste sentido Mirwald et al. ${ }^{4}$ desenvolveram uma técnica prática e não invasiva, que requer uma avaliação única de poucas variáveis antropométricas, capaz de predizer a distância em anos em que um indivíduo se encontra da sua idade do PVC.

Usando os sincronismos diferenciais conhecidos do crescimento da estatura, da altura tronco-cefálica e dos membros inferiores, é possível pressupor que as relações proporcionais de mudança entre esses segmentos, podem prover uma indicação do status maturacional ${ }^{4}$.

Nesse modelo, foram incluídas interações entre comprimento de perna e altura tronco-cefálica, idade e comprimento de pernas, idade e altura tronco-cefálica, bem como razão entre peso e estatura. A partir desses dados obtidos originalmente de numa população canadense, e da posterior replicação do estudo em amostras canadenses e belgas, foi proposto um modelo mais "abrangente", a partir da combinação das três populações, sendo sugerido como o mais indicado por sua característica generalista, descrito abaixo:

\section{Para rapazes}

$$
\begin{aligned}
\mathrm{PVC}= & -9,236+0,0002708(\mathrm{CPxTC})- \\
& -0,001663(\mathrm{IxCP})+0,007216(\mathrm{IxTC}) \\
& +0,02292(\mathrm{P} / \mathrm{E})
\end{aligned}
$$

Onde: $\mathrm{CP}=$ Comprimento de Perna; $\mathrm{TC}=$ Altura TroncoCefálica; $\mathrm{I}=\mathrm{Idade} ; \mathrm{P}=$ Peso; $\mathrm{E}=$ Estatura

Aceitáveis índices de determinação $\left(\mathrm{r}^{2}=0,89\right)$ e erro padrão da estimativa $(\mathrm{EPE}=0,569)$ foram encontrados ${ }^{4}$. Além do mais, o nível de precisão do modelo tem sido investigado em outros estudos, uma vez que é possível sua aplicação em diferentes delineamentos de pesquisa-11.

A utilização de instrumentos para avaliação da maturação biológica, que sejam eficientes e de fácil aplicação pode auxiliar na correta interpretação da maturação relacionada ao desempenho motor. Todavia, a indicação da melhor alternativa deve ser investigada, uma vez que seus efeitos podem contribuir para a elaboração de um plano didático-metodológico que norteiem os exercícios para jovens esportistas. Sendo assim, o objetivo do estudo foi comparar a relação entre o desempenho motor e diferentes formas de classificação da maturação biológica, envolvendo um grupo de jovens brasileiros, praticantes de futebol.

\section{PROCEDIMENTO METODOLÓGICO}

A amostra foi composta por 209 sujeitos do sexo masculino, com idades entre 6 e 17 anos, participantes de atividades oferecidas em um centro esportivo para a prática de futebol. Neste local funciona o Projeto Betesda, patrocinado pela Secretaria Municipal de Esportes de Presidente Prudente/SP, onde são oferecidas além das atividades de iniciação e treinamento da modalidade, aulas de inglês, informática e educação moral.

O estudo seguiu as diretrizes e normas que regulamentam a pesquisa com seres humanos 
(lei 196/96). O projeto que envolve este estudo foi submetido ao Comitê de Ética e Pesquisa da FCT-UNESP, sob Protocolo de Pesquisa $\mathrm{n}^{\circ} 180 / 2007$, tendo sido aprovado em agosto de 2007. Após obtenção de termo de consentimento livre e esclarecido dos participantes e autorização dos pais ou responsável, os dados foram coletados.

\section{Indicadores de Maturação biológica}

A Idade (milesimal) foi determinada calculando-se a fração milesimal, estimada de acordo com o método desenvolvido por Healy, citado e adaptado por Guedes e Guedes ${ }^{12}$. Para efeito de categorização etária, uma vez determinada a idade milesimal, os intervalos para cada idade foram determinados para aqueles sujeitos que estivessem entre - 0,500 a 0,499 do valor inteiro de cada idade. Por exemplo: a idade de 10 anos compreendeu por todos que tivessem idade milesimal entre 9,500 a 10,499 anos.

Os sujeitos foram classificados, também, mediante auto-avaliação em indicações em figuras, dos estágios de maturação sexual de acordo com os critérios definidos por Tanner ${ }^{13}$ dos estágios do desenvolvimento dos pêlos e das genitálias. Foi estimada também a idade do $\mathrm{PVC}^{4}$, a partir das medidas requeridas pelo modelo apresentado anteriormente. Uma vez que esse modelo retorna valores contínuos e a maturação para efeito descritivo é categorizada em valores inteiros, para efeito descritivo, esses valores foram aproximados aos inteiros mais próximos. Por ser Yi o nível de maturação para o i-ésimo indivíduo, a classificação foi definida em oito níveis (-4 a 3 anos) na forma expressa na Tabela 1.

\section{Antropometria}

As variáveis antropométricas foram mensuradas segundo a padronização da International Society for Advancement in Kinanthropometry - ISAK ${ }^{14}$. As medidas de peso corporal foram realizadas em balança mecânica da marca Fillizola, com precisão de 100 gramas; a estatura e a altura tronco-cefálica foram mensuradas com o auxílio de um estadiômetro de madeira, com escala de precisão de $0,1 \mathrm{~cm}$, sendo esta última, obtida em medida tríplice coincidente, uma vez que se tratou da medida mais solicitada nas interações da fórmula preditiva do PVC. O comprimento de membros inferiores foi encontrado pela diferença entre a altura tronco-cefálica e estatura.
Tabela 1. Classificação do Pico de Velocidade de Crescimento.

\begin{tabular}{cc}
\hline Nível & Intervalo (anos) considerado \\
\hline-4 & $Y_{i}<-3,49$ \\
-3 & $-3,50 \leq Y_{i}<-2,50$ \\
-2 & $-2,50 \leq Y_{i}<-1,50$ \\
-1 & $-1,50 \leq Y_{i}<-0,50$ \\
0 & $-0,50 \leq Y_{i}<0,50$ \\
1 & $0,50 \leq Y_{i}<1,50$ \\
2 & $1,50 \leq Y_{i}<2,50$ \\
3 & $Y_{i} \geq 2,50$ \\
\hline
\end{tabular}

\section{Desempenho motor}

As variáveis do desempenho motor foram obtidas através dos protocolos sugeridos pela American Alliance for Health, Physical Education, Recreation and Dance ${ }^{15}$. Foram realizados os testes de sentar-e-alcançar, salto horizontal, abdominal modificado, corrida de 50 metros e corrida/caminhada de 9/12 minutos.

Um teste de força de preensão manual foi administrado utilizando-se um dinamômetro manual rusSO ДИНАМОМЕТР КИСТЕВОЙ ДК modelo AK 140 com precisão de 2 daN, sendo os valores convertidos em kilograma força. $\mathrm{O}$ avaliado realizou duas tentativas, com intervalos de 60 segundos na posição em pé, braços estendidos ao longo do corpo, sendo registrado o maior valor da mão direita e esquerda, sucessivamente. Os dados foram coletados sempre pelos mesmos avaliadores, treinados nos testes e integrantes da equipe de avaliação de um grupo de estudos da FCT-Unesp, Presidente Prudente/SP. Os valores de reprodutibilidade das medidas estiveram sempre dentro dos limites convencionais.

\section{Tratamento estatístico}

Para o tratamento das informações e descrição dos resultados, os sujeitos foram agrupados pela idade do pico de velocidade de crescimento (PVC). Nas comparações descritivas, o cálculo das medidas de tendência central (média e desvio padrão) foi utilizado para caracterizar os grupos. Uma análise exploratória prévia foi empregada para detectar a existência de outliers (não encontrados) e o teste de normalidade de Kolmogorov-Smirnov foi efetuado, indicando que os dados apresentaram tendência à normalidade. A seguir, efetuou-se uma análise de variância (ANOVA - one way) a um fator (PVC), quando o teste Post-hoc de Tukey localizou as diferenças entre os grupos.

Os testes de correlação (Pearson e Spear- 
Tabela 2. Média, desvio padrão (dp) e análise de variância de caracterização, antropometria e desempenho motor, de diferentes idades biológicas (PVC) em jovens futebolistas de seis a 17 anos de idade.

\begin{tabular}{|c|c|c|c|c|c|c|c|}
\hline \multirow{2}{*}{ PVC } & $-4(n=72)$ & $-3(n=51)$ & $-2(n=27)$ & $-1(n=28)$ & $0(n=17)$ & $1(n=9)$ & $2(n=5)$ \\
\hline & $\begin{array}{l}\text { Média } \\
\text { (dp) }\end{array}$ & $\begin{array}{l}\text { Média } \\
\text { (dp) }\end{array}$ & $\begin{array}{l}\text { Média } \\
\text { (dp) }\end{array}$ & $\begin{array}{l}\text { Média } \\
\text { (dp) }\end{array}$ & $\begin{array}{l}\text { Média } \\
\text { (dp) }\end{array}$ & $\begin{array}{c}\text { Média } \\
\text { (dp) }\end{array}$ & $\begin{array}{l}\text { Média } \\
\text { (dp) }\end{array}$ \\
\hline \multicolumn{8}{|l|}{ Caracterização } \\
\hline Idade (anos) & $\begin{array}{c}8,90 \\
(1,37)\end{array}$ & $\begin{array}{l}11,36 \\
(0,96)\end{array}$ & $\begin{array}{l}12,48 \\
(0,75)\end{array}$ & $\frac{13,86}{(0,76)}$ & $\begin{array}{l}14,76 \\
(0,75)\end{array}$ & $\begin{array}{l}15,78 \\
(0,83)\end{array}$ & $\frac{16,80}{(0,45)}$ \\
\hline \multicolumn{8}{|l|}{ Antropometria } \\
\hline Estatura (cm) & $\begin{array}{c}132,98 \\
(7,03)\end{array}$ & $\begin{array}{l}146,0 \\
(5,28)\end{array}$ & $\begin{array}{c}153,84 \\
(4,89)\end{array}$ & $\begin{array}{c}163,65 \\
(6,66)\end{array}$ & $\frac{170,65}{(7,28)}$ & $\begin{array}{c}175,19 \\
(6,88)\end{array}$ & $\frac{182,32}{(5,86)}$ \\
\hline Peso (Kg) & $\begin{array}{l}29,08 \\
(5,76)\end{array}$ & $\frac{37,68}{(7,05)}$ & $\begin{array}{l}43,04 \\
(8,84)\end{array}$ & $\frac{52,41}{(10,21)}$ & 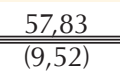 & $\frac{70,02}{(10,58)}$ & $\begin{array}{l}62,28 \\
(4,74)\end{array}$ \\
\hline TR-C (cm) & $\begin{array}{l}69,60 \\
(3,33)\end{array}$ & $\begin{array}{l}74,75 \\
(2,78)\end{array}$ & $\begin{array}{l}78,93 \\
(3,04)\end{array}$ & $\begin{array}{l}83,38 \\
(3,25)\end{array}$ & $\frac{87,17}{(2,71)}$ & 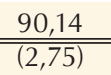 & $\frac{93,76}{(2,14)}$ \\
\hline $\mathrm{Mi}(\mathrm{cm})$ & $\begin{array}{l}63,38 \\
(5,66)\end{array}$ & $\begin{array}{l}71,25 \\
(4,16)\end{array}$ & $\begin{array}{l}74,92 \\
(3,00)\end{array}$ & $\begin{array}{l}80,27 \\
(5,03)\end{array}$ & 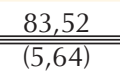 & $\begin{array}{l}85,04 \\
(5,01)\end{array}$ & $\begin{array}{l}88,56 \\
(4,54)\end{array}$ \\
\hline \multicolumn{8}{|c|}{ Desempenho Motor } \\
\hline FMD (Kg) & $\begin{array}{c}13,38 \\
(3,4)\end{array}$ & $\begin{array}{l}17,71 \\
(3,19)\end{array}$ & $\begin{array}{l}21,26 \\
(4,23)\end{array}$ & $\frac{27,79}{(6,58)}$ & $\underline{30,94}$ & $\frac{36,0}{(6,84)}$ & $\begin{array}{l}36,80 \\
(4,60)\end{array}$ \\
\hline FME (Kg) & $\begin{array}{l}12,77 \\
(3,92)\end{array}$ & $\frac{17,72}{(3,08)}$ & $\frac{20,19}{(4,71)}$ & $\begin{array}{l}26,79 \\
(6,71)\end{array}$ & $\begin{array}{l}29,71 \\
(6,02)\end{array}$ & 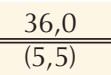 & 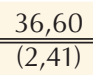 \\
\hline $\mathrm{SA}(\mathrm{cm})$ & $\frac{24,19}{(6,07)}$ & 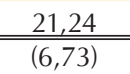 & 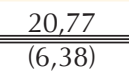 & 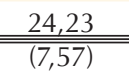 & 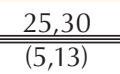 & $\frac{25,88}{(10,96)}$ & 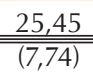 \\
\hline $\mathrm{SH}(\mathrm{cm})$ & $\begin{array}{c}129,24 \\
(20,9)\end{array}$ & $\frac{146,14}{(29,34)}$ & $\frac{149,7}{(27,57)}$ & $\frac{172,63}{(23,17)}$ & $\begin{array}{l}174,71 \\
(23,51)\end{array}$ & $\begin{array}{l}188,50 \\
(17,05)\end{array}$ & $\frac{189,4}{(13,83)}$ \\
\hline AB (rep.) & $\frac{24,03}{(7,67)}$ & 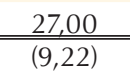 & $\frac{30,33}{(7,22)}$ & $\frac{34,11}{(\overline{(7,23)}}$ & $\underline{35,65}$ & 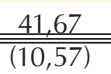 & $\frac{39,0}{(5,94)}$ \\
\hline $50 \mathrm{~m}(\mathrm{~m} / \mathrm{s})$ & $\frac{5,28}{(4,93)}$ & $\begin{array}{c}5,68 \\
(5,49)\end{array}$ & $\frac{5,89}{(0,44)}$ & $\frac{6,41}{(0,49)}$ & $\frac{6,48}{(0,49)}$ & $\frac{6,81}{(0,48)}$ & $\frac{7,07}{(0,72)}$ \\
\hline 9/12 $\min .(\mathrm{m} / \mathrm{min})$ & $\frac{129,20}{(46,87)}$ & $\frac{106,70}{(48,23)}$ & $\frac{99,23}{(60,25)}$ & $\frac{123,04}{(96,18)}$ & $\frac{130,10}{(100,07)}$ & $\frac{194,04}{(20,72)}$ & $\frac{218,95}{(29,33)}$ \\
\hline
\end{tabular}

${ }^{1} \mathrm{Tr}-\mathrm{C}=$ Altura tronco-cefálica; $\mathrm{Mi}=$ altura dos membros inferiores; FMD = Força da mão direita; FME = Força da mão esquerda; $\mathrm{SA}=$ Sentar-e-Alcançar; $\mathrm{SH}=$ Salto horizontal; $\mathrm{AB}=$ Abdominal modificado; $50 \mathrm{~m}=$ Corrida de 50 metros; 9/12 min. = Corrida de 9 ou 12 minutos.

${ }^{2}$ As faixas etárias sublinhadas pela mesma linha $\underline{\mathrm{NÃO}}$ diferem estatisticamente $(\mathrm{p}<0,05)$.

man) entre os instrumentos de classificação da maturação biológica e desempenho motor foram empregados para testar as relações entre as variáveis, apontando aquelas de maior magnitude. Uma vez que os maiores coeficientes de correlação entre desempenho motor e estado maturacional foram observados no PVC, seguiu-se uma análise de regressão múltipla para identificar a magnitude dessas relações. Todas as análises foram realizadas através do pacote estatístico SPSS 13.0 for Windows, adotando-se o nível de significância estatística menor ou igual a 5\%.

\section{RESULTADOS}

Uma vez que o modelo de Mirwald et al. ${ }^{4}$ para o PVC retorne um valor contínuo, optou-se pela representação dos resultados por agrupamento etário do PVC, possibilitando as comparações discretas entre os demais indicadores de matu- ração biológica.

A evolução da caracterização, antropometria e desempenho motor pela idade do PVC dos sujeitos e a ANOVA (one-way) estão representados na Tabela 2. O comportamento dos valores de média e desvio padrão (dp) dos dados indica que houve uma tendência de aumento concorrente à aproximação ou ultrapassagem do PVC.

Observou-se, ainda, que as maiores variações do desempenho motor (desvios padrão) ocorreram próximo do PVC, no intervalo entre menos e mais um ano. A análise de variância a um fator (PVC) indicou diferenças entre os grupos maturacionais (Tukey) na maioria das comparações. As exceções foram marcadas com uma linha contínua (Tabela 2). Todavia nota-se uma maior similaridade na maioria das variáveis dos valores médios do PVC -1 a 2, sugerindo maior homogeneidade, principalmente na antropometria) à medida que se 
Tabela 3. Correlação nível de significância entre indicadores de classificação biológica (Idade, PVC, Genitálias e Pêlos) e Desempenho Motor de jovens futebolistas de seis a 17 anos de idade.

\begin{tabular}{llccc}
\hline \multirow{2}{*}{ Desempenho Motor } & \multicolumn{2}{c}{ Pearson } & \multicolumn{2}{c}{ Spearman } \\
\cline { 2 - 5 } & Idade & PVC & $0,63^{*}$ & $0,72^{*}$ \\
\hline Força Mão Direita (Kg) & $0,77^{*}$ & $0,86^{*}$ & $0,64^{*}$ & $0,73^{*}$ \\
Força Mão Esquerda (Kg) & $0,77^{*}$ & $0,85^{*}$ & 0,02 & 0,08 \\
Sentar e Alcançar (cm) & 0,06 & 0,09 & $0,54^{*}$ & $0,61^{*}$ \\
Salto Horizontal (cm) & $0,64^{*}$ & $0,67^{*}$ & $0,47^{*}$ & $0,50^{*}$ \\
Abdominais (rep.) & $0,52^{*}$ & $0,53^{*}$ & $0,62^{*}$ & $0,67^{*}$ \\
Corrida 50m (m/s) & $0,71^{*}$ & $0,73^{*}$ & $0,38^{*}$ & $0,44^{*}$ \\
Corrida 9/12 min (m/min) & $0,47^{*}$ & $0,50^{*}$ & \\
\hline
\end{tabular}

$*(p<0,05)$

Tabela 4. Modelos de regressão linear 'Stepwise' na determinação das variáveis de desempenho motor, a partir de indicadores de classificação biológica (Idade, PVC, Pêlos e Genitálias) em jovens futebolistas de seis a 17 anos de idade.

\begin{tabular}{|c|c|c|c|c|c|c|}
\hline $\begin{array}{l}\text { Variável D } \\
\text { ependente }\end{array}$ & & $\mathrm{R}$ & $\mathrm{R}^{2}$ ajustado & EPE & $\begin{array}{c}\text { Variáveis } \\
\text { Independentes }\end{array}$ & $\begin{array}{c}\text { Coeficiente } \\
\text { e Beta }\end{array}$ \\
\hline \multirow[b]{2}{*}{ Salto Horizontal } & Modelo 1 & 0,68 & 0,46 & 21,39 & $\begin{array}{l}\text { Intercepto } \\
\text { PVC }\end{array}$ & $\begin{array}{c}177,84 \\
0,65\end{array}$ \\
\hline & Modelo 2 & 0,68 & 0,47 & 21,19 & $\begin{array}{l}\text { Intercepto } \\
\text { PVC } \\
\text { Pilosidade }\end{array}$ & $\begin{array}{c}160,58 \\
0,53 \\
0,18\end{array}$ \\
\hline Abdominais & Modelo 1 & 0,53 & 0,28 & 7,92 & $\begin{array}{l}\text { Intercepto } \\
\text { PVC }\end{array}$ & $\begin{array}{c}35,99 \\
0,53\end{array}$ \\
\hline \multirow[b]{2}{*}{ Corrida 50m } & Modelo 1 & 0,73 & 0,53 & 0,49 & $\begin{array}{l}\text { Intercepto } \\
\text { PVC }\end{array}$ & $\begin{array}{l}6,55 \\
0,73\end{array}$ \\
\hline & Modelo 2 & 0,75 & 0,55 & 0,48 & $\begin{array}{l}\text { Intercepto } \\
\text { PVC } \\
\text { Pilosidade }\end{array}$ & $\begin{array}{l}6,02 \\
0,55 \\
0,23\end{array}$ \\
\hline Corrida 9/12min. & Modelo 1 & 0,51 & 0,25 & 28,86 & $\begin{array}{l}\text { Intercepto } \\
\text { PVC }\end{array}$ & $\begin{array}{c}187,13 \\
0,51\end{array}$ \\
\hline \multirow{2}{*}{$\begin{array}{l}\text { Dinamometria } \\
\text { (mão direita) }\end{array}$} & Modelo 1 & 0,86 & 0,74 & 4,38 & $\begin{array}{l}\text { Intercepto } \\
\text { PVC }\end{array}$ & $\begin{array}{c}30,64 \\
0,86\end{array}$ \\
\hline & Modelo 2 & 0,87 & 0,76 & 4,19 & $\begin{array}{l}\text { Intercepto } \\
\text { PVC } \\
\text { Idade (anos) }\end{array}$ & $\begin{array}{r}56,91 \\
1,36 \\
-0,52\end{array}$ \\
\hline \multirow{3}{*}{$\begin{array}{l}\text { Dinamometria } \\
\text { (mão esquerda) } \\
\text { Corrida 9/12min. }\end{array}$} & Modelo 1 & 0,86 & 0,74 & 4,35 & $\begin{array}{l}\text { Intercepto } \\
\text { PVC }\end{array}$ & $\begin{array}{c}29,98 \\
0,86\end{array}$ \\
\hline & Modelo 2 & 0,87 & 0,76 & 4,19 & $\begin{array}{l}\text { Intercepto } \\
\text { PVC } \\
\text { Idade (anos) }\end{array}$ & $\begin{array}{c}54,40 \\
1,32 \\
-0,49\end{array}$ \\
\hline & Modelo 3 & 0,88 & 0,76 & 4,14 & $\begin{array}{c}\text { Intercepto } \\
\text { PVC } \\
\text { Idade (anos) } \\
\text { Pelos }\end{array}$ & $\begin{array}{c}49,91 \\
1,21 \\
-, 048 \\
0,14\end{array}$ \\
\hline
\end{tabular}

$\beta=$ Beta; $R=$ Coeficiente de correlação; $E P E=E$ rro padrão da estimativa; $p(F)=$ significância de $F$ ajustado; Limites (IC)=Intervalo de confiança.

atinge a maturidade biológica.

A Tabela 3 apresenta os valores encontrados para os coeficientes de correlação e os níveis de significância entre idade, índices de maturação e as variáveis de desempenho motor.

Os coeficientes obtidos no PVC indicaram maior robustez na relação com todas as variáveis de desempenho motor do que os observados com idade, genitália ou pêlos, quando a correlação variou de moderada a alta na maioria das comparações. Nota-se que somente a variável flexibilidade obtida pelo teste de sentar-e-alcançar, não alcançou significância estatística em nenhuma forma de classificação biológica.

$\mathrm{Na}$ tentativa de identificar a participação das variáveis de classificação biológica e sua participação no desempenho motor dos jovens futebolistas, foi realizada uma análise de regressão linear (Stepwise). Cada teste foi considerado como variável dependente, e os indicadores maturacionais entraram no modelo como variáveis independentes, como podem ser vistos na Tabela 4. Não foi possível 
ser efetuada a regressão entre o teste Sentar e Alcançar e as variáveis de classificação biológica, dada à baixa relação entre elas. Nas demais comparações, a análise resultou de 1 a 3 modelos por variável de desempenho motor.

Em todos os modelos de todas as variáveis de desempenho motor, o PVC entrou como primeira variável preditiva. Nos testes de Abdominais e corrida de 9/12 minutos, foi a única variável capaz de explicar a variabilidade dos resultados.

No primeiro exemplo do Modelo 1 da tabela, o PVC foi capaz de explicar sozinho 46\% da variância do salto horizontal. Em conjunto com os pêlos pubianos (Modelo 2) o poder de explicação aumentou em apenas 1\%. Esta mesma tendência se seguiu em todas as demais comparações, com poder de explicação do desempenho motor pelo PVC variando entre $25 \%$ e $74 \%$.

Embora nos segundos modelos o PVC continuasse sendo a principal componente de explicação do desempenho motor, a pilosidade pubiana teve a segunda maior participação no salto horizontal, na corrida de 50 metros e como última variável no Modelo 3 da dinamometria da mão esquerda. A idade entrou nos segundos modelos de dinamometria das mãos direita e esquerda, porém, curiosamente, os valores foram negativos, sugerindo que a força de preensão manual depende de outros fatores que não da idade.

\section{DISCUSSÃO}

Neste estudo, as relações entre o desempenho motor, idade, PVC e maturação sexual foram consideradas. A idade biológica pode ser avaliada por vários métodos, incluindo critérios sexuais, morfológicos, dentais e esqueléticos. Embora nenhum sistema da maturação biológica forneça uma descrição completa da maturação de um indivíduo, as inter-relações entre os sistemas são, não obstante, fortes o bastante para indicar os níveis biológicos do desenvolvimento de um grupo de crianças ou adolescentes ${ }^{8}$.

As comparações entre os testes de desempenho motor e os indicadores de maturação biológica sugeriram efeitos de diferentes intensidades, segundo a forma de classificação (Idade, PVC, pêlos e genitálias) utilizada. A forma descritiva dos resultados classificados pelo PVC (Tabela 2) indicou uma seqüência progressiva em todas as variáveis envolvidas no estudo, não apontando as oscilações comumente observadas em descri- ções das variáveis motoras por idade ${ }^{3,16,17}$.

Isto já sugere uma tendência de progressão lógica mais realista do desenvolvimento antropométrico e motor, quando a classificação dos jovens é ordenada pelo PVC. Resultados semelhantes também foram observados por Lefevre et al..$^{18}$, ao estudarem longitudinalmente a performance motora relacionada ao PVC de 173 rapazes belgas por cerca de 6 anos.

Embora no presente estudo não se tenha controlado a maturação precoce ou tardia, os resultados indicam que o desvio padrão (dp) das idades em cada nível de PVC foi menor que um ano na maioria dos casos. No nível -4 , todavia, observou-se a maior abrangência etária, sugerindo que o ponto mais longe de atingir o PVC contém o grupo mais heterogêneo. A média etária do PVC, no presente estudo (14,76 anos), foi ligeiramente maior em relação às obtidas em estudos longitudinais (14,22 - Beunen et al. ${ }^{19}$; 14,32 - Lefreve et al. $\left.{ }^{18}\right)$ ou transversais $(13,40$ - Iuliano-Burns et al..$^{20} ; 13,72$ - Sherar et al. ${ }^{9}$ ), dando a entender a interferência de fatores étnicos e regionais.

As observações do presente estudo indicaram maior consistência na classificação biológica pelo PVC do que em outras formas de classificação maturacional. Com exceção do teste sentar-e-alcançar, a análise de correlação entre maturação biológica e desempenho motor (Tabela 3), resultou nos maiores índices de determinação (valores apresentados em $\mathrm{r}^{2}$ ) para a variável PVC $(0,25$ a 0,74$)$ do que genitálias $(0,14$ a 0,41$)$, pêlos pubianos $(0,19$ a 0,53$)$ ou idade $(0,22$ a 0,59). Por essa razão, o PVC tem sido sugerido como uma medida ideal para alinhar os participantes de acordo com um marcador maturacional comum ${ }^{8,11}$, como, por exemplo, em comparações entre sexos.

Beunen et al. ${ }^{19}$ relataram coeficientes de determinação $\left(\mathrm{r}^{2}\right)$ capazes de explicar apenas de 4\% a 36\% da variância entre maturação esquelética e desempenho motor em rapazes de 12 a 19 anos de idade. Jones et al. ${ }^{2)}$ também obtiveram coeficientes de determinação não tão altos em rapazes $(0,31$ a 0,53$)$ e menores ainda em moças $(0,06$ a 0,21) classificados por maturação sexual. Mesmo assim, esses autores recomendam fortemente que no estudo do desempenho motor, particularmente para meninos, deve-se fazer exame da maturidade biológica.

Nos estudos citados acima, os coeficientes de correlação foram relativamente menores que 
no presente estudo. Apesar de escassos, quando os estudos utilizaram maturação por PVC, como Lefreve et al..$^{18}$ que observaram variáveis de força explosiva, força estática, velocidade do movimento dos membros superiores, os coeficientes de correlação foram moderados e altos $(0,47$ a 0,83), claramente relacionados ao status de maturação (PVC). Todavia, esses autores lembram que é difícil concluir que esta é a causa da influência da maturação sobre o desempenho motor, pois outros fatores como estatura e peso podem confundir os resultados.

Embora um fator geral da maturidade possa esboçar o tempo do crescimento e da maturação durante a adolescência, em ambos os sexos, há uma variação suficiente para sugerir que nenhum sistema (i.e., sexual, esquelético ou somático) fornece uma descrição completa do tempo da maturação durante a adolescência. As correlações entre os indicadores são geralmente médias a elevadas, mas tendem a ser mais baixas para meninos do que para meninas ${ }^{8}$.

Sherar et al. ${ }^{9}$ obtiveram correlação baixa a moderada $\left(r^{2}=0,30-0,55, p<0,05\right)$ entre PVC e a idade em que se alcançam cada estágio da pilosidade púbica. Mas com maiores coeficientes $\left(r^{2}=0,69\right)$ com maturação esquelética ${ }^{11}$. A aparente discórdia entre os indicadores acima mencionados reflete a variação individual no sincronismo e no tempo da maturidade sexual e somático e os interesses metodológico de cada estudo ${ }^{8}$.

De acordo com os resultados obtidos pela análise de regressão linear (Stepwise), considerando os valores de $r^{2}$ ajustado, verificou-se que somente os primeiros modelos (intercepto e PVC) das variáveis de desempenho motor, foram capazes de explicar entre $25 \%$ e $74 \%$ (média $=50 \%$ ) do desempenho obtido nos testes motores (Tabela 4). Quando considerada a classificação pelo PVC mais a variável pilosidade, puderam ser explicados $47 \%$ a $76 \%$ dos resultados de três testes do desempenho motor, e $76 \%$ para PVC e idade em dois testes. Estes resultados são bem maiores que os obtidos em comparações etárias ${ }^{16,17}$, sexuais ${ }^{8,10}$, esqueléticas ${ }^{8}$ ou somáticas ${ }^{4,23}$.

Os resultados observados neste estudo sugerem alta associação entre o PVC e o desempenho motor uma vez que os efeitos do tamanho corporal são em parte considerados na determinação do PVC. Porém, é fortemente recomendado que essa relação seja mais bem investigada, uma vez que fatos limitantes neste estudo como: a) o reduzido número de indivíduos; b) esta amostra ser composta de sujeitos do sexo masculino, praticantes de uma única modalidade esportiva; c) a falta de comparação entre maturação precoce, normal e tardia; d) e a não consideração do tamanho corporal (segmentar e dimensional), não permitem afirmar que a maturação biológica seja a causa exclusiva dos efeitos sobre o desempenho motor destes jovens.

Baxter-Jones et al. ${ }^{17}$ lembram que deve se considerar o processo do crescimento e da maturação normais antes que uma conclusão definitiva possa ser alcançada. A menos que o tamanho do corpo e a maturidade biológica sejam considerados, nenhum deles pode isoladamente identificar os efeitos independentes da atividade ou do treinamento físico.

Todavia a facilidade e rapidez na obtenção da medida do PVC, não requerendo sofisticados equipamentos nem alta treinabilidade dos avaliadores ${ }^{4}$, certamente são qualidades desejáveis em estudos populacionais. É possível ainda classificar maturação precoce, normal e tardia pelo $\mathrm{PVC}^{20}$. Sobretudo, a subjetividade de classificação biológica é minimizada mediante obtenção mais precisa das medidas necessárias para sua determinação ${ }^{17}$, como é o caso do PVC. Além do mais, esse recurso possibilita utilização em diversas configurações: na forma original de estimativa para a idade (contínua) do PVC, em agrupamentos etários (como neste estudo) ou como indicador do marco maturacional $(\mathrm{PVC}=0)$ em pré e pós $\mathrm{PVC}^{19}$.

\section{CONCLUSÃO}

Sendo assim, os resultados encontrados neste estudo mostram que, quando foi considerada a idade do PVC, foi possível encontrar uma melhor explicação para o desempenho em testes motores, segundo o status maturacional, superando os valores encontrados nas comparações por idade cronológica ou maturação sexual (genitálias e pêlos).

Por se tratar de um método não invasivo, de fácil obtenção e que não requer alta treinabilidade, o uso do PVC apresenta vantagens éticas na classificação da maturação biológica de crianças e adolescentes. Sobretudo, por sua alta relação com o desempenho motor, muito embora nas idades finais do PVC essas diferenças tenderam a diminuir.

Assim, a classificação da maturação pelo 
PVC isoladamente pôde explicar uma maior parcela dos resultados obtidos nos testes de desempenho motor, quando comparados com diferentes formas de classificação da maturação biológica. Dessa forma, a utilização do PVC como instrumento de classificação biológica passa a ser, preferencialmente, recomendada para jovens com características semelhantes às da amostra deste estudo.

\section{REFERÊNCIAS BIBLIOGRÁFICAS}

1. Matsudo VKR, Matsudo SMM. Avaliação e prescrição de atividade física na criança. Rev da APEF - Londrina 1995;10(17):46-55.

2. Malina RM, Bouchard C, Bar-Or O. Growth, maturation and physical activity. 2.ed. Champaign, IL: Human Kinetics; 2004.

3. Malina RM, Bouchard C. Growth, maturation and physical activity. Champaign: Human Kinetics; 1991. p. 70-83.

4. Mirwald RL, Baxter-Jones ADG, Bailey DA, Beunen GP. An assessment of maturity from anthropometric measurements. Med Sci Sports Exerc 2002;34(4):689-694.

5. Bergmann GG, Bergmann MLA, Lorenzi TDC, Pinheiro ES, Garlipp DC, Moreira RB, et al. Pico de velocidade em estatura, massa corporal e gordura subcutânea de meninos e meninas dos 10 aos 14 anos de idade. Rev Bras Cineantropom Desempenho Hum 2007;9(4):333-338.

6. Guedes DP. Implicações no estudo da composição corporal. In: Amadio AC, Barbanti VJ. A biodinâmica do movimento humano e suas relações interdisciplinares. São Paulo: Estação Liberdade; 2000.

7. Machado DRL. Maturação esquelética e desempenho motor em crianças e adolescentes. [Dissertação de Mestrado em Educação Física]. São Paulo (SP): Universidade de São Paulo; 2004.

8. Baxter-Jones ADG, Eisenmann JC, Sherar LB. Controlling for maturation in pediatric exercise science. Pediatr Exerc Sci 2005;17(1):18-30.

9. Sherar LB, Baxter-Jones AD, Mirwald RL. Limitations to the use of secondary sex characteristics for gender comparisons. Ann Hum Biol 2004;31(5):586-593.

10. Sherar LB, Mirwald RL, Baxter-Jones AD, Thomis M. Prediction of adult height using maturitybased cumulative height velocity curves. J Pediatr 2005;147(4):508-514.

11. Matthews BL, Bennell KL, Mckay HA, Khan KM, Baxter-Jones AD, Mirwald RL, et al. The influence of dance training on growth and maturation of young females: a mixed longitudinal study. Ann Hum Biol 2006;33(3):342-356.
12. Guedes DP, Guedes JERP. Manual prático para avaliação em educação física. Barueri: Manole; 2006.

13. Tanner JM. Growth at adolescence, 2nd ed. Oxford, UK:Blackwell Scientific Publications; 1962.

14. Norton K, Olds T, editors. Antropométrica. Porto Alegre: Artmed; 2005.

15. American Alliance for Health, Physical Education, Recreation and Dance. Health Related Physical Fitness Technical Manual. Reston: AAHPERD; 1984.

16. Kemper HC, De Vente W, Van Mechelen W, Twisk JW. Adolescent motor skill and performance: is physical activity in adolescence related to adult physical fitness? Am J Hum Biol 2001;3(2):180189.

17. Malina RM. Physical activity and fitness: childhood to adolescence to adulthood. Am J Hum Biol 2001;13(2):162-172.

18. Lefreve J, Beunen G, Steens G, Claessens A, Renson R. Motor performance during adolescence and age thirty as related to age at peak height velocity. Ann Hum Biol 1990;17(5):423-435.

19. Beunen GP, Malina RM, Lefevre J, Claessens AL, Renson R, Simons J. Prediction of adult stature and noninvasive assessment of biological maturation. Med Sci Sports Exerc 1997;29(2):225-230.

20. Iuliano-Burns S, Mirwald, RL, Bailey DA. Timing and magnitude of peak height velocity and peak tissue velocities for early, average, and late maturing boys and girls. Am J Human Biol 2001(1);13:1-8.

21. Jones MA, Hitchen PJ, Straton G. The importance of considering biological maturation when assessing physical fitness measures in girls and boys aged 10 to 16 years. Ann Hum Biol 2000;27(1):57-65.

22. Wainer H, Roche AF, Bell S. Predicting adult stature without skeletal age and without parental data. Pediatrics 1978;61(4):569-572.

23. Roche AF, Wainer H, Thissen D. The RWT method for the prediction of adult stature. Pediatrics 1975;56(6):1026-1033. 